\title{
Characteristics of patients admitted to in-patient psychiatric care following parasuicide
}

\author{
Polash Shajahan, Katharine Logan and George Masterton
}

\begin{abstract}
Parasuicide referrals and subsequent transfers to psychiatric in-patient care were recorded for January to April, 1972 to 1996. Information regarding the psychiatric outcome of transfers for the years 1985, 1990 and 1994 was obtained by case note review. Parasuicide referrals show a decline from 1972 to 1990 but they have more than doubled since then. Referrals transferred to in-patient poychiatric care fell from 10.7 to $2.8 \%$ during 1985 to 1994 . Fewer potients with acute stress reaction, adjustment disorder, personality disorder and substance misuse are being transferred. Transfers of potients with 'severe mental illness' have remained constant.
\end{abstract}

Studies in Scotland have shown that the number (and rates) of parasuicide admissions to general hospitals declined during the late 1970s and throughout the 1980s, then increased after 1990 (Platt et al, 1988; Stark et al, 1994; McLoone \& Crombie, 1996). The proportion of male admissions has increased (Stark et al, 1994), as has the proportion using paracetamol (Hawton \& Fagg, 1992; McLoone \& Crombie, 1996). The trend towards deinstitutionalisation and reduction of in-patient psychiatric beds has meant that greater numbers of people with mental disorder are now being treated in the community. This group of people are inherently more vulnerable to stress, self-harming behaviour (parasuicide) and completed suicide. In recent years it has been argued that recognising and treating mental illness is a more viable option than attempts at directly preventing suicide (Wilkinson, 1994). Psychiatric consultation appears to have a positive effect on outcome following parasuicide (Soukas \& Lonnqvist, 1991). Hawton et al (1993) argue that specifically targeting young people with substance misuse after parasuicide may prevent their suicide. It is possible that a subgroup of patients who are admitted to psychiatric care following parasuicide are a particularly high group for which psychiatric intervention and resources should be targeted (Nordentoft et al, 1993; Gunnell et al, 1995).

\section{The study}

The number of cases of parasuicide referred to our parasuicide assessment service for the first four months of the years 1972 to 1996 and the proportion transferred to in-patient psychiatric care were examined. The characteristics of patients newly transferred to in-patient psychiatric care (i.e. those not already psychiatric inpatients), in the years 1985, 1990 and 1994 were examined in detail. The reason for choosing these years is that they span a decade of radical changes in the delivery of mental health services. During this period the number of psychiatric beds was reduced (1028 in 1985 to 728 in 1994, Royal Edinburgh Hospital figures). We hypothesised that (a) the proportion of patients transferred to in-patient psychiatric care will fall due to the trend in caring for people in the 'community' and the reduced availability of in-patient beds, and that (b) the number of patients with severe mental health problems transferred will increase as a result of shorter in-patient admissions and inadequacies in community care.

Information concerning the referral of patients to the parasuicide assessment service and subsequent transfer to psychiatric in-patient care was obtained from records kept since the late 1960s. Between 1967 and 1990 standardised data were collected on all parasuicide patients presenting to the Royal Infirmary of Edinburgh (Kreitman, 1977; Platt et al, 1988). Around $85 \%$ of attenders at the accident and emergency (A\&E) department were admitted to the Regional Poisoning Treatment Centre, $90 \%$ of whom were then assessed by psychiatrists. Those not admitted or assessed were predominantly self-discharges, and their proportion of $20-25 \%$ of the total number of presentations remained constant. Routine data collection ceased in 1990, so there is no way of knowing whether there has been a change in these figures. However, the system of assessment and the policy for admission of parasuicide patients has not changed since 1990. 


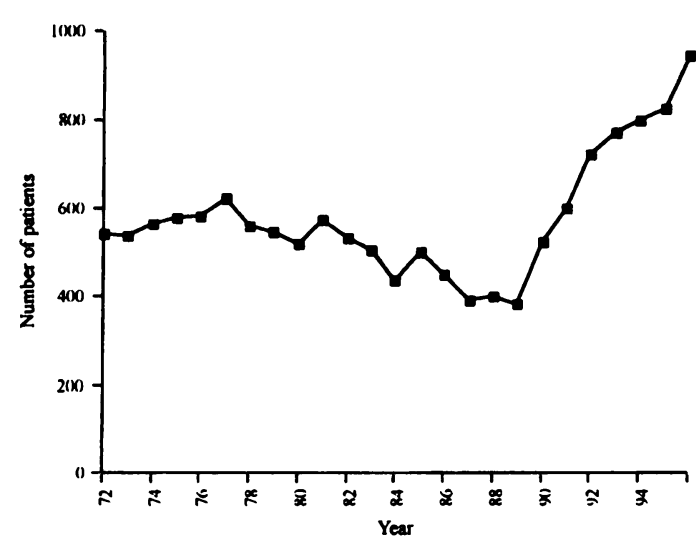

Figure 1. Number of parasuicide admissions undergoing psychiatric assessment

Information regarding the psychiatric admission was obtained from retrieval of psychiatric case notes. The following variables were examined: age, marital status, duration of in-patient stay, use of compulsory admission, follow-up provision, psychiatric readmission within one year and diagnoses. In an attempt to reduce bias, as high a proportion of case notes as possible were retrieved $(86,85$ and $87 \%$ were achieved for the years 1985, 1990 and 1994 respectively). There is insignificant within year variation in the number of parasuicides presenting locally during this part of the year (Masterton, 1991). Some patients being transferred to in-patient psychiatric care were already psychiatric in-patients: this subgroup was included in the percentages transferred to psychiatric care but not considered in subsequent analyses. Case note recording of diagnoses had been standardised locally to the International Classification of Diseases (ICD-9) (World Health Organization, 1978) across the three sample years. Age and duration of inpatient stay were normally distributed and parametric tests ( $t$-test) were used to analyse these. All other variables were analysed using non-parametric tests (chi-squared).

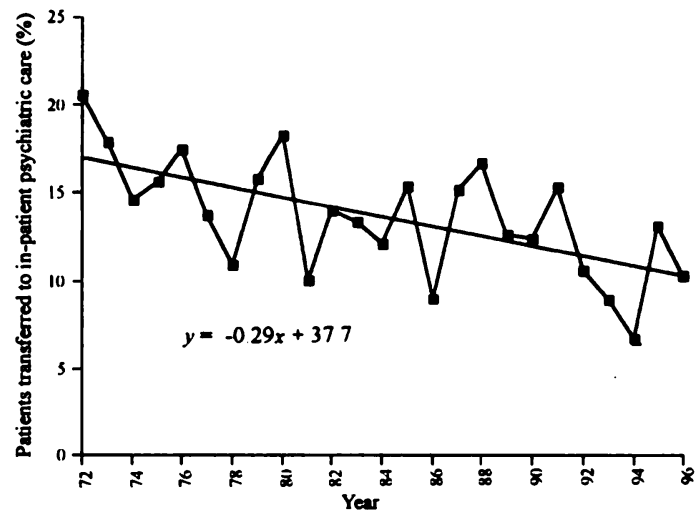

Figure 2. Percentage of parasuicide patients transferred to in-patient psychiatric care

\section{Findings}

Figure 1 displays the number of parasuicide referrals to psychiatry in the first four months of each year for the years 1972 to 1996 . A decline during the 1980 s was followed by a sharp increase continuing through to 1996, with a doubling of referrals during the 1990s.

Figure 2 presents the percentage of all patients who were transferred to in-patient psychiatric care. There is a significant negative correlation between the year and the percentage of all patients transferred $(r=-0.63, P<0.01)$. Over the past 25 years there has been a decline in the percentage of transfers from 17 to $10 \%$ of cases referred.

Table 1 shows referral and transfer details of all patients presenting to the parasuicide assessment service. The total number of parasuicide referrals had increased from 503 in 1985 to 799 in 1994. The total number of transfers had fallen (71 to 41 ), as had the number (54 to 23 ) and percentage (10.7 to 2.8$)$ of new transfers. The number of existing in-patients transferred back has remained relatively constant.

Table 2 shows demographic and clinical details of patients newly transferred to psychiatric

Table 1. Referral and transfer details (\%) of all patients transferred to psychiatric in-patient care following parasuicide

\begin{tabular}{llll}
\hline & 1985 & 1990 & 1994 \\
\hline Total number of referrals & 503 & 525 & 799 \\
Total number of transfers & $71(14)$ & $55(10.5)$ & $41(5)$ \\
Number of new transfers & $54(10.7)$ & $34(6.5)$ & $23(2.8)$ \\
Number of existing in-patients transferred back & $17(3.4)$ & $21(4)$ & $18(2.3)$ \\
\hline
\end{tabular}


Table 2. Demographic and clinical details of patients newly transferred to psychiatric in-patient care following parasuicide

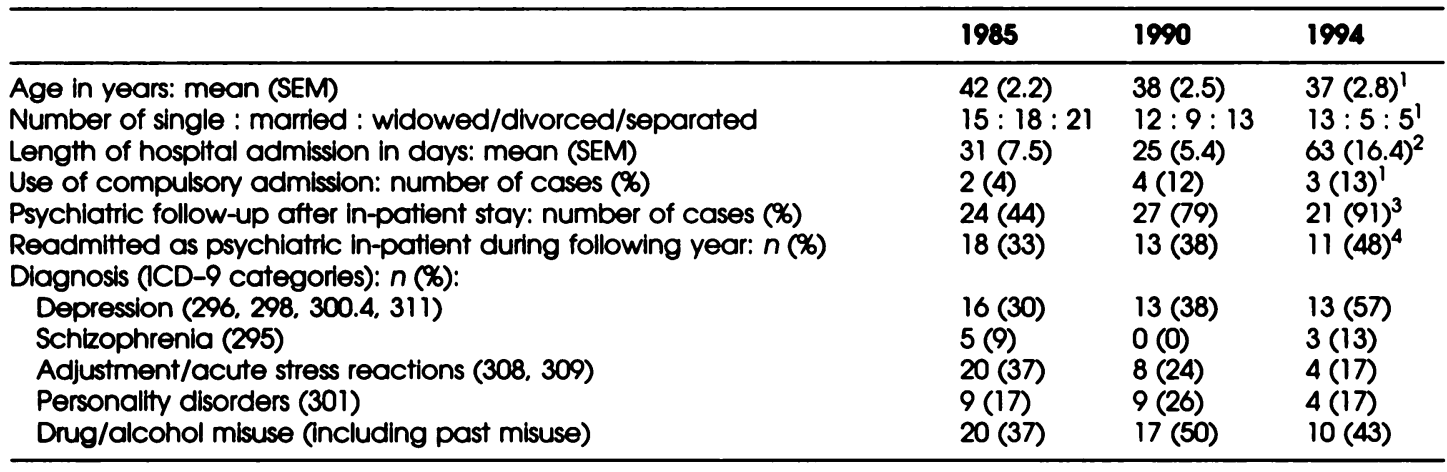

1. Not significant.

2. $t=-2.03$, d.f. $=75, P<0.05$.

3. $\chi^{2}=19.27$, d.f. $=2, P<0.001$.

4. $\chi^{2}=8.25$, d.f. $=1, P<0.005$ (males).

in-patient care following parasuicide: there were no significant gender differences; the mean age of patients has remained constant; marital status has not changed significantly; the average length of hospital admission has increased significantly, almost doubling in 1994 compared to 1985 and 1990; and the use of compulsory admission is uncommon and has not changed significantly. Psychiatric follow-up is more likely in 1994 compared to previous years, as is the likelihood of psychiatric readmission within one year for males.

The number of patients with a final diagnosis of depression was relatively constant, although it represented an increasing proportion of transfers. Less patients with final diagnoses of adjustment disorder, acute stress reactions, personality disorders and drug or alcohol misuse were being transferred.

\section{Comments}

In keeping with other studies (Stark et al, 1994; McLoone \& Crombie, 1996) there was a decline in parasuicide activity presenting to our service over the period 1972 to 1990 , followed by a sharp increase continuing through to 1996 . It is not possible to conclude that this reflects a true change in the epidemiology of parasuicide because other factors may be contributing, for example, altered clinical practice (Stark et al 1994). In a previous Edinburgh study it was found that $20 \%$ of parasuicide patients were treated by their general practitioner and not assessed in hospital (Kreitman, 1977). It has not been established whether this percentage has changed. Kreitman's (1977) study showed that $10 \%$ of parasuicide patients in Edinburgh presented to other general hospitals, although $40 \%$ of these were subsequently transferred to the Royal Infirmary. The closure of the A\&E Department at the Western General Hospital (Edinburgh) in 1990 left the Royal Infirmary as the only unit accepting parasuicide admissions; this change probably accounts for 5-10\% of the increase observed.

Our first hypothesis is supported in that the proportion of patients newly transferred to inpatient psychiatric care has fallen from 10.7 to $2.8 \%$ of all referrals. There are two possible explanations for this: first, the number of inpatient beds for both acute and long-stay patients over the past decade has decreased so that admission is increasingly confined to patients who have more serious mental illness as opposed to acute stress or adjustment disorders; second, the development of other options such as day care provision and community psychiatric teams.

Our second hypothesis is not supported. While the proportion of transfers of patients with serious mental illnesses has indeed substantially increased, the overall numbers in these categories has remained constant. If community care for the seriously mentally ill is breaking down then a likely consequence would be increased suicidal behaviour in these patients. Our study does not answer this important question but we can say that there is no evidence of increasing numbers of these former patients being readmitted to psychiatric wards following a parasuicide admission.

Although it seems unlikely that a breakdown in community care was responsible for the changes we observed over the decade, the role of the psychiatric hospital has been changing in 
keeping with Wessely's observations (1996), in that psychiatric wards are no longer acting as places of asylum or providing 'time-out' for patients who have acute stress or adjustment disorders. We no longer are obliged and/or have the option of transferring patients in crisis who are not mentally ill, while the patients who are transferred are a population that require considerable input from psychiatric services. Whether these changes have been beneficial to needy patients with what are considered to be less severe forms of mental disorder requires investigation.

\section{Acknowledgements}

We thank the staff of Ward 22, Royal Infirmary of Edinburgh for their cooperation and the medical records staff of the Royal Edinburgh and associated hospitals for their help in retrieving case notes.

\section{References}

Gunnel. D. J., Peters, T. J., Kammerung. R. M.. et al (1995) Relation between parasuicide. sulcide. psychiatric admissions. and socioeconomic deprivation. British Medical Joumal, 311. 226-230.

HAWTON. K. \& FAGG. J. (1992) Trends in deliberate selfpoisoning and self-injury in Oxford, 1976-90. British Medical Journal, 304, 1409-1411.

-. - Platt. S., et al (1993) Factors associated with suicide after parasuicide in young people. British Medical Journal, 306. 1641-1644.

KRETTMAN. N. (ed.) (1977) Parasuicide. London: Wiley.

MASTERTON, G. (1991) Monthly and seasonal variation in parasuicide. A sex difference. British Joumal of Psychiatry. 168. 155-157.
MCLOONE, P. \& CROMBIE, I. K. (1996) Hospitalisation for deliberate self-polsoning in Scotland from 1981 to 1993: trends in rates and types of drugs used. British Journal of Psychiatry, 169, 81-85.

NORDENTOFT, M., BREUM, L., MUNCK, L. K., et al (1993) High mortality by natural and unnatural causes; a 10 year follow-up study of patients admitted to a poisoning treatment centre after suicide attempts. British Medical Journal, 306, 1637-1641.

Platt. S., HaWton, K., KRETMAan, N., et al (1988) Recent clinical and epidemiological trends in parasuicide in Edinburgh and Oxford: a tale of two cities. Psychological Medicine, 18, 405-418.

SOUKAS, J. \& LONNgVIST, J. (1991) Outcome of attempted suicide and psychiatric consultation: risk factors and suicide mortality during a five-year follow-up. Acto Psychiatrica Scandinavica, 84, 545-549.

STARK, C.. SMTTH, M. \& HALL. D. (1994) Increase in parasuicide in Scotland. British Medical Joumal, 308. 1569-1570.

WESSELY, S. (1996) The rise of counselling and the return of alienism. British Medical Joumal, 313, 158-160.

WILKINSON, G. (1994) Can suicide be prevented? British Medical Journal, 309, 860-862.

WORLD HEALTH ORGaNization (1978) Mental Disorders: Glossary and Guide to their Classification in Accordance with the Ninth Revision of the International Classification of Diseases. Geneva: WHO.

*Polash Shajahan, Formerly Registrar, Alcohol Problems Clinic, Royal Edinburgh Hospital; Katharine Logan, Research Registrar, Royal Edinburgh Hospital; and George Masterton, Consultant Psychiatrist, Royal Infirmary of Edinburgh

"Correspondence: Dr Shajahan. Ward 1, Royal Edinburgh Hospital, Morningside Terrace, Edinburgh EH10 5HF 